\title{
PRODUTIVIDADE DE FITOMASSA DE REBROTAS DE SORGO EM CONSÓRCIO COM CAPIM-PAIAGUÁS
}

\author{
ALLAN HISASHI NAKAO ${ }^{1}$, MARCELO ANDREOTTI ${ }^{2}$, VIVIANE CRISTINA MODESTO', \\ ISABÔ MELINA PASCOALOTO ${ }^{3}$ e DEYVISON DE ASEVEDO SOARES ${ }^{1}$
}

\author{
'Programa de Pós-Graduação em Sistema de Produção, Universidade Estadual Paulista “Júlio de Mesquita Filho”, \\ Departamento de Fitossanidade, Engenharia Rural e Solos, Ilha Solteira, São Paulo, Brasil. \\ allanhisashinakao@gmail.com; vivianemodesto@hotmail.com;deyvison_a.soares@hotmail.com \\ ${ }^{2}$ Docente, Universidade Estadual Paulista “Júlio de Mesquita Filho”, \\ Departamento de Fitossanidade, Engenharia Rural e Solos, Ilha Solteira, São Paulo, Brasil. dreotti@agr.feis.unesp.br \\ ${ }^{3}$ Programa de Pós-Graduação em Agricultura, Universidade Estadual Paulista “Júlio de Mesquita Filho”, \\ Botucatu, São Paulo, Brasil. isabomelina@gmail.com
}

Revista Brasileira de Milho e Sorgo, v.18, n.1, p. 133-147, 2019

RESUMO: O objetivo do trabalho foi avaliar o desempenho produtivo de matéria seca e verificar o acúmulo de nutrientes das rebrotas do sorgo granífero ou dupla aptidão consorciados ou não com capim-paiaguás, com ou sem a inoculação por Azospirillum brasilense, para continuidade do sistema plantio direto no Cerrado, após colheita para ensilagem. O experimento foi realizado na Fazenda de Ensino, Pesquisa e Extensão da Universidade Estadual Paulista (Unesp) - Campus de Ilha Solteira, localizada no município de Selvíria-MS. O delineamento experimental foi em esquema fatorial $2 \times 2 \times 2$, com quatro repetições, sendo os tratamentos constituídos por dois anos de cultivo (2015 e 2016); em cultivo exclusivo (solteiro) dos sorgos (granífero ou dupla aptidão) ou em consórcio com o capim-paiaguás, com ou sem a inoculação das sementes de sorgo com a bactéria diazotrófica Azospirillum brasilense. O aproveitamento da rebrota do sorgo granífero ou de dupla aptidão, principalmente em consórcio com o capim-paiaguás, apresentou-se viável na produção de palhada remanescente no período de entressafra, pela maior produtividade de matéria seca, com maior acúmulo de nutrientes.

Palavras-chave: Decomposição, integração lavoura-pecuária, Urochloa brizantha, plantio direto.

\section{PHYTOMASS PRODUCTIVITY OF SORGHUM INTERCROPPED WITH PAIAGUÁS GRASS}

\begin{abstract}
This study objectified to evaluate the dry matter productive performance and to verify the nutrient accumulation of sorghum regrowths with or without Urochloa brizantha cv. BRS Paiaguás (paiaguás grass) intercropped, and with or without Azospirillum brasilense inoculation, for the continuity of the no-tillage system in the Cerrado, after harvesting for ensiling. The experiment was carried out at the Teaching, Research and Extension Farm of the Universidade Estadual Paulista (UNESP) - Ilha Solteira Campus, located in the municipality of Selvíria, Mato Grosso do Sul State. The experimental design used a 2 × 2 × 2 factorial scheme, with four replications. Treatments were two years of cultivation (2015 and 2016) and two different seeding arrangements of sorghum (grain or dual aptitude), monocropped or intercropped with paiaguás grass, with or without inoculation of sorghum seeds with Azospirillum brasilense. The use of regrowth of both sorghum types (grain sorghum or dual aptitude), mainly intercropped with paiaguás grass, was viable for the production of remaining straw in the off-season period, due to the higher productivity of dry matter, with greater accumulation of nutrients.
\end{abstract}

Keywords: Decomposition, crop-livestock integration, Urochloa brizantha, no-tillage. 
Nos sistemas integrados de produção agropecuária, o sorgo vem sendo cultivado nas áreas de Cerrado pela adaptação da cultura a situações em que o déficit hídrico e as condições de baixa fertilidade dos solos oferecem maiores riscos para outras culturas como o milho safrinha.

O sorgo é o quinto cereal mais produzido no mundo, depois de milho, trigo, arroz e cevada (FAO, 2014), visando à produção de feno, silagem, grãos ou pasto (Botelho et al., 2010). Na safra 2016/2017, estimou-se uma área cultivada de 628,5 mil hectares no Brasil, sendo concentrada na região Centro-Oeste (Acompanhamento da Safra Brasileira [de] Grãos, 2017), pela aptidão a solos de Cerrado, tolerância à seca e menor custo de produção quando comparado ao milho. A produção é majoritariamente destinada à alimentação animal em razão da sua aceitabilidade, grande valor nutritivo e produtividade (Conab, 2011).

Neste sentido, o sorgo tem ganhado espaço nos últimos anos nas áreas de Cerrado, por causa, principalmente, das características de resistência à seca e da alta capacidade de rebrota, o que permite que mesmo após colheita dos grãos e/ou material vegetal para ensilagem deixe palhada para continuidade do sistema plantio direto. Segundo Zago (1991), após a colheita da cultura original do sorgo, a planta conserva vivo seu sistema radicular, possibilitando sua rebrota, desde que haja condições de temperatura, umidade no solo e fertilidade adequada.

O sorgo em consórcio com as espécies do gênero Urochloa vem obtendo resultados positivos (Silva et al., 2013) em sistemas de produção integrados. Entretanto, na tecnologia em consorciar culturas deve-se atentar ao fato de que as cultivares apresentam diferentes alturas de plantas, uma vez que na competição entre plantas estão atreladas umas às outras. Assim, o sorgo granífero tem menor estatura e, por- tanto, menor capacidade competitiva com capins em consórcio, quando comparado ao sorgo de dupla aptidão ou forrageiro, de maior estatura e com provável maior efeito competitivo com forrageiras consorciadas. Em contrapartida, o sorgo de dupla aptidão ou mesmo o forrageiro apresenta maior produtividade de forragem do que o granífero, entretanto, com menor proporcionalidade de grãos, que tem influência direta na qualidade da forragem e/ou material a ser ensilado.

A importância da cobertura do solo por restos culturais de rebrotas de sorgo e capins do gênero Urochloa é identificada como alternativa econômica de rotação de culturas no sistema plantio direto por produzir alta quantidade de matéria seca, para posterior decomposição e liberação de nutrientes, resultando em boa proteção do solo por períodos de tempo mais prolongados por causa da sua alta relação $\mathrm{C} / \mathrm{N}$.

Além disso, é crescente a procura por novas tecnologias que visem aumento da produção e a manutenção da sustentabilidade nos sistemas agrícolas de produção. A inoculação das sementes de sorgo com Azospirillum brasilense tem sido amplamente utilizada, além de aumentar a disponibilidade de nitrogênio através da fixação biológica de nitrogênio, seu efeito hormonal pode aumentar a tolerância da planta ao estresse hídrico (Dartora et al., 2013).

Assim, o objetivo do trabalho foi avaliar a produtitividade de matéria seca e verificar o acúmulo de nutrientes das rebrotas do sorgo granífero ou dupla aptidão consorciado ou não com capim-paiaguás com ou sem a inoculação por Azospirillum brasilense, para continuidade do sistema plantio direto no Cerrado.

\section{Material e Métodos}

O experimento foi desenvolvido na Fazenda de Ensino, Pesquisa e Extensão da Faculdade de Enge- 
nharia/Unesp - Campus de Ilha Solteira, localizada no município de Selvíria-MS (20²0’05" S e 51²4'26" WGr e aproximadamente $335 \mathrm{~m}$ de altitude). Apresenta $1.370 \mathrm{~mm}$ de precipitação pluvial média anual, $23,5{ }^{\circ} \mathrm{C}$ de temperatura média anual e umidade relativa do ar média de 64,8\%. Na Figura 1 estão apresentados os dados de precipitação pluvial, temperatura máxima, média e mínima durante o período de condução do experimento. O clima da região é Aw, segundo classificação de Köppen, e caracterizado como tropical úmido com estação chuvosa no verão e seca no inverno. O solo da área experimental, segundo o Sistema Brasileiro de Classificação de Solos (Santos et al., 2013), é um Latossolo Vermelho Distrófico típico argiloso.

Antes da instalação dos experimentos, coletaram-se vinte amostras de solo nas camadas de 0,0-0,10 e 0,10-0,20 m, as quais foram submetidas a análises químicas para fins de fertilidade do solo, seguindo a metodologia proposta por Raij et al. (2001), e foram obtidos os seguintes valores dos atributos químicos na camada de $0,0-0,10 \mathrm{~m}: \mathrm{pH}\left(\mathrm{CaCl}_{2}\right)=5,4 ; 16,0 \mathrm{mg}$ $\mathrm{dm}^{-3}$ de P; 1,6; 27,0; 19,0; 28,0; 47,6 e 75,6 mmol $_{\text {c }}$ $\mathrm{dm}^{-3}$ de $\mathrm{K}, \mathrm{Ca}, \mathrm{Mg}, \mathrm{H}+\mathrm{Al}, \mathrm{SB}$ e CTC, respectivamente; $24,0 \mathrm{~g} \mathrm{dm}^{-3}$ de matéria orgânica e saturação por bases $(\mathrm{V} \%)=63,0$, e para a camada de $0,10-0,20 \mathrm{~m}$ : $\mathrm{pH}\left(\mathrm{CaCl}_{2}\right)=5,5 ; 17,0 \mathrm{mg} \mathrm{dm}^{-3}$ de P; 1,2; 25,0; 16,0; 28,0; 42,2 e 70,2 $\mathrm{mmol}_{\mathrm{c}} \mathrm{dm}^{-3}$ de $\mathrm{K}, \mathrm{Ca}, \mathrm{Mg}, \mathrm{H}+\mathrm{Al}$, $\mathrm{SB}$ e CTC, respectivamente; $20,0 \mathrm{~g} \mathrm{dm}^{-3}$ de matéria orgânica e V\%=60,0.

Em 17/03/2015 e 06/04/2016, respectivamente, ocorreram as semeaduras dos sorgos granífero (cv. Ranchero) e de dupla aptidão (cv. A 9902). Os experimentos foram implantados no mesmo local em ambos os anos, e o objetivo final foi a produção de material para silagem. O delineamento experimental foi em

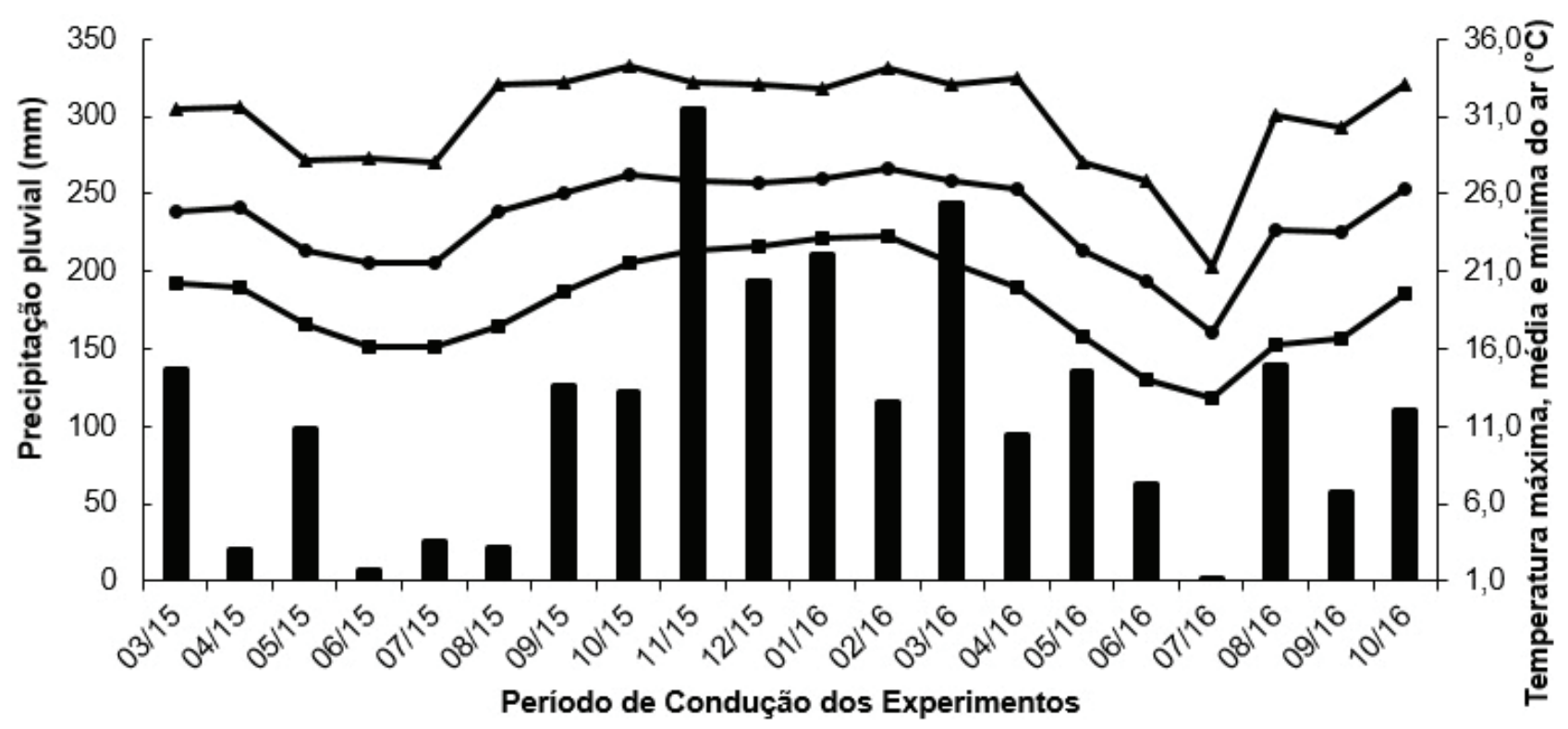

$\longrightarrow$ Precip. Total $(\mathrm{mm}) \rightarrow$ Tmédia $\left({ }^{\circ} \mathrm{C}\right) \longrightarrow$ Tmáx. $\left({ }^{\circ} \mathrm{C}\right) \rightarrow$ Tmín. $\left({ }^{\circ} \mathrm{C}\right)$

Figura 1. Precipitação pluvial e temperaturas máxima, média e mínima obtidas da estação meteorológica situada na Fazenda de Ensino, Pesquisa e Extensão da FE/Unesp, no município de Selvíria, no Mato Grosso do Sul, no período de março/2015 a outubro de 2016. 
blocos casualizados, em esquema fatorial $2 \times 2 \times 2$, com quatro repetições, sendo os tratamentos constituídos por dois anos de cultivo (2015 e 2016); em cultivo exclusivo (solteiro) do sorgo ou em consórcio com o capim-paiaguás (Urochloa brizantha cv. BRS Paiaguás), com ou sem a inoculação das sementes de sorgos com a bactéria Azospirillum brasilense.

$\mathrm{Na}$ semeadura do sorgo, utilizou-se semeadora-adubadora com mecanismo sulcador tipo haste (facão) para sistema plantio direto (SPD), no espaçamento de 0,45 m, com densidade de 10 sementes $\mathrm{m}^{-1}$. Na sequência foi semeado o capim-paiaguás com outra semeadora-adubadora com mecanismo sulcador do tipo disco duplo desencontrado para SPD. As sementes foram depositadas na profundidade de aproximadamente $0,06 \mathrm{~m}$, no espaçamento de $0,45 \mathrm{~m}$, utilizando-se cerca de $10 \mathrm{~kg} \mathrm{ha}^{-1}$ de sementes puras viáveis com valor cultural $(\mathrm{VC})=60 \%$, nos dois anos de condução. A bactéria diazotrófica foi fornecida pelo inoculante líquido (estirpes Ab-V5 e Ab-V6) na dose de $100 \mathrm{~mL} 25 \mathrm{~kg}$ de sementes ${ }^{-1}$. A inoculação foi efetuada momentos antes da semeadura, à sombra, e nas sementes das cultivares de sorgo nos dois anos.

Após a colheita do material vegetal para ensilagem (10/06/2015 e 12/07/2016) na altura de 0,25 $\mathrm{m}$ do solo, a área foi vedada por 99 dias para o crescimento das rebrotas do sorgo e/ou capim colhidos, as quais foram colhidas em 16/09/2015 e 19/10/2016, simulando o período de vedação de uma pastagem. Nestas datas coletaram-se as plantas, utilizando o método do quadrado de metal $(1,0 \times 1,0 \mathrm{~m})$, com 3 repetições por parcela. Posteriormente as amostras foram separadas em cada fração vegetal, pesadas e colocadas em estufa de ventilação forçada a $65{ }^{\circ} \mathrm{C}$ por $72 \mathrm{~h}$ para determinação da quantidade de cada fração (colmos, folhas e panículas e de capim quando em consórcio), que somadas resultaram na produti- vidade de massa seca total de forragem (sorgo e/ou capim). Destas amostras foi retirada uma quantidade de material para determinação dos teores de N, P, K, $\mathrm{Ca}, \mathrm{Mg}$ e S, segundo a metodologia proposta por Malavolta et al. (1997), visando cálculo do acúmulo de macronutrientes que poderiam, pela decomposição da palhada, liberar gradativamente esses nutrientes, para a continuidade do sistema plantio direto. Após esse procedimento, a área foi dessecada e roçada.

Os resultados foram submetidos à análise de variância pelo teste $\mathrm{F}$ e as médias foram comparadas pelo teste de Tukey a 5\% de probabilidade com auxílio do programa estatístico SISVAR $®$ (Ferreira, 2014).

\section{Resultados e Discussão}

Nas Figuras 2 e 3 estão apresentados os resultados obtidos para produtividade de matéria seca das rebrotas do sorgo granífero e de dupla aptidão, respectivamente. Para os sorgos granífero e dupla aptidão, as produtividades foram superiores no segundo ano de cultivo (Figura 2A e 3A), pela maior precipitação pluvial neste ano (Figura 1) e possivelmente pela melhoria dos atributos físicos e químicos do solo proporcionados pelo primeiro ano de condução do experimento. Esse resultado demonstra que mesmo exportando grandes quantidades de matéria seca e nutrientes por conta de duas safras de silagens, os sistemas produtivos apresentaram-se como técnicas viáveis, proporcionando o bom estabelecimento das forrageiras, seja em função do consórcio ou da inoculação com bactérias diazotróficas.

Os resultados observados neste trabalho são numericamente inferiores aos encontrados por Botelho et al. (2010), que obtiveram produtividades de 12,$3 ; 12,7 ; 9,5$ e $9,3 \mathrm{t} \mathrm{ha}^{-1}$ de MS total nas rebrotas, 
trabalhando com os genótipos de sorgo Volumax, BRS 610, AG 2005E e Qualimax, respectivamente. Entretanto, neste caso todas as cultivares testadas são forrageiras e cultivadas na safra de primavera/verão.

Normalmente, as cultivares de sorgo, após o primeiro corte, têm a capacidade de rebrote, o que caracteriza estas plantas como excelentes opções para o cultivo em propriedades rurais visando o fornecimento de palhada residual para a continuidade do SPD. Entretanto, quando em consórcio com espécies forrageiras, como as do gênero Urochloa, como realizado, a competição com o capim-paiaguás após o corte, diminuiu a capacidade de rebrote do sorgo. Verificou-se que, após a colheita das culturas para ensilagem, o sorgo pouco se desenvolveu, dando lugar para o crescimento do capim-paiaguás que foi implantado em consórcio (Figura 2B).

A inoculação de sementes de sorgo realizada na semeadura proporcionou efeito residual com maior produção de matéria seca da rebrota em todos os componentes morfológicos do sorgo e do capim quando em consórcio (MSFG-R, MSCG-R, MSPG-R, MSTG -R e PMSTG-R - Figura 2C). Esses resultados podem ser atribuídos tanto ao efeito da fixação biológica do nitrogênio, quanto aos mecanismos de promoção de crescimento, que maximizam o volume radicular e a capacidade de as plantas absorverem e assimilarem nutrientes do solo (Dobbelaere et al., 2001). Em trabalho realizado utilizando-se inoculação de $A$. brasilense via foliar no sorgo granífero no primeiro corte, Nakao et al. (2014) constataram maiores massas secas para colmos, folhas e panículas nos tratamentos inoculados com as bactérias diazotróficas.

No desdobramento da interação entre anos de cultivo, com ou sem o capim-paiaguás (Tabela 1), para produtividade de matéria seca das folhas e total do sorgo das rebrotas, observou-se que o sorgo se sobressaiu quando não houve consorciação e sempre no segundo ano de cultivo. Assim, ficou evidente que há consorciação do capim-paiaguás com o sorgo granífero na safrinha existe redução de seu crescimento por conta da competição, fato este mais expressivo com limitação hídrica (primeiro ano). No entanto, para continuidade do SPD, cujo intuito é fornecer mais palhada ao sistema, a consorciação com capim -paiaguás é viável (Figura 2B) como demostrado na produtividade de matéria seca total das rebrotas do sorgo granífero (MSTG-R).

Na Tabela 2 do desdobramento entre anos de cultivo, com ou sem inoculação, para MSFG-R, MSCG-R, MSPG-R e MSTG-R, fica evidente o efeito da bactéria nas rebrotas do sorgo granífero, onde provavelmente o A. brasilense contribuiu com o crescimento vegetal, pela intensificação da produção de hormônios, e também contribuiu para promover a fixação biológica do $\mathrm{N}_{2}$, primordial ao rebrote das plantas. Segundo Hungria (2011), a forma com maior potencial de quebrar a tripla ligação que une os dois átomos de nitrogênio atmosférico $\left(\mathrm{N}_{2}\right)$ é pela ação da enzima nitrogenase dos microrganismos.

Na Figura 3 constam as produtividades de matéria seca das rebrotas do sorgo de dupla aptidão para os diferentes tratamentos. Constata-se que o segundo ano foi o que mais se destacou na produção de massa seca (forrageira + sorgo de dupla aptidão) com um total de 3,66 tha ${ }^{-1}$, sendo superior ao primeiro ano $(1,35$ $\left.\mathrm{t} \mathrm{ha}^{-1}\right)$. Esse comportamento para altas produtividades no segundo ano pode estar relacionado às maiores precipitações pluviais (Figura 1), melhorando seu desenvolvimento, como também observado no sorgo granífero. Segundo Magalhães e Durães (2003), plantas em condições de estresse hídrico, tendem a diminuir o seu metabolismo, enrolando suas folhas e cessando seu crescimento como mecanismo de defe- 


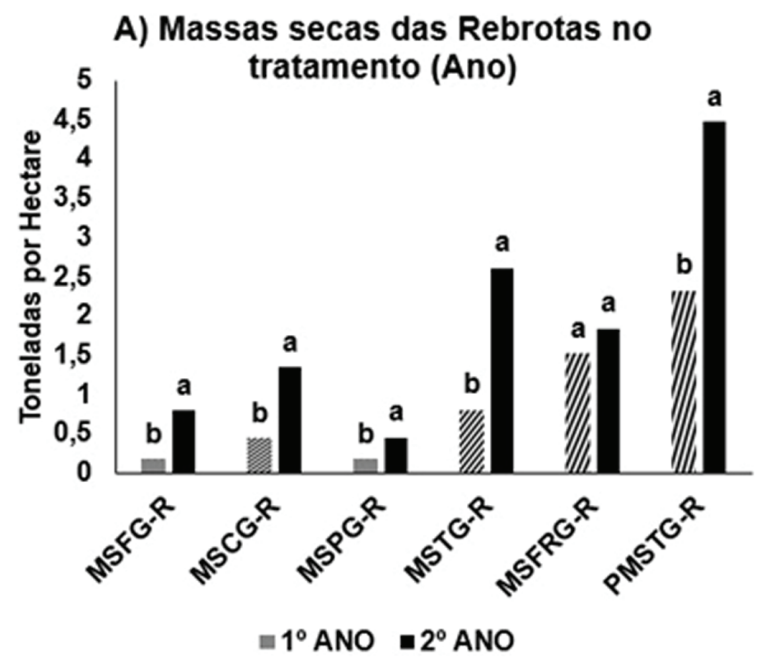

\begin{tabular}{lrr}
\hline Fator & \multicolumn{1}{c}{$\mathrm{Fc}$} & $\mathrm{Pr}>\mathrm{Fc}$ \\
\hline Ano (MSFG-R) & 98 & 0,001 \\
\hline Ano (MSCG-R) & 99,25 & 0,001 \\
\hline Ano (MSPG-R) & 37,29 & 0,001 \\
\hline Ano (MSTG-R) & 95,05 & 0,001 \\
\hline Ano (MSFRG-R) & 0,28 & 0,28 \\
\hline Ano (PMSTG-R) & 74,46 & 0,001 \\
\hline
\end{tabular}

\section{B) Massas secas das Rebrotas no}

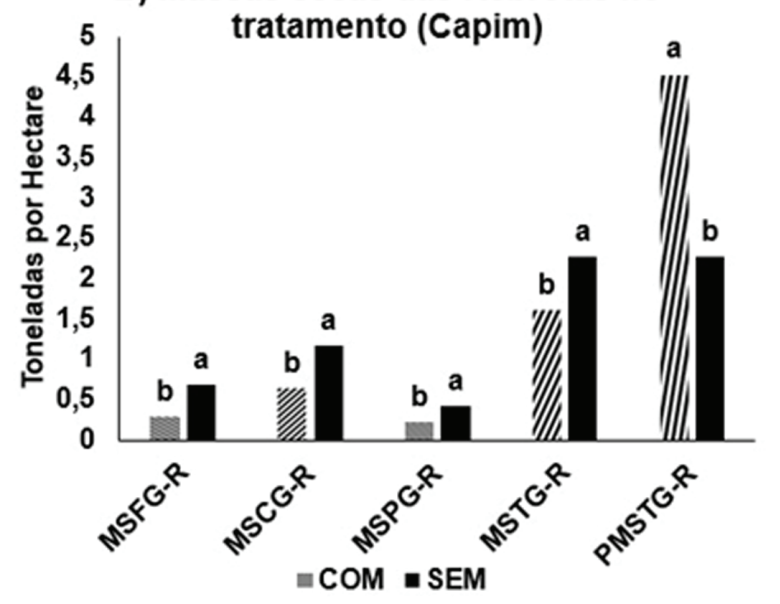

\begin{tabular}{lrr}
\hline Fator & $\mathrm{FC}$ & $\mathrm{Pr}>\mathrm{FC}$ \\
\hline Capim (MSFG-R) & 57,8 & 0,001 \\
\hline Capim (MSCG-R) & 34,25 & 0,001 \\
\hline Capim (MSPG-R) & 19,56 & 0,001 \\
\hline Capim (MSTG-R) & 40,94 & 0,001 \\
\hline Capim (PMSTG-R) & 83,2 & 0,001 \\
\hline
\end{tabular}

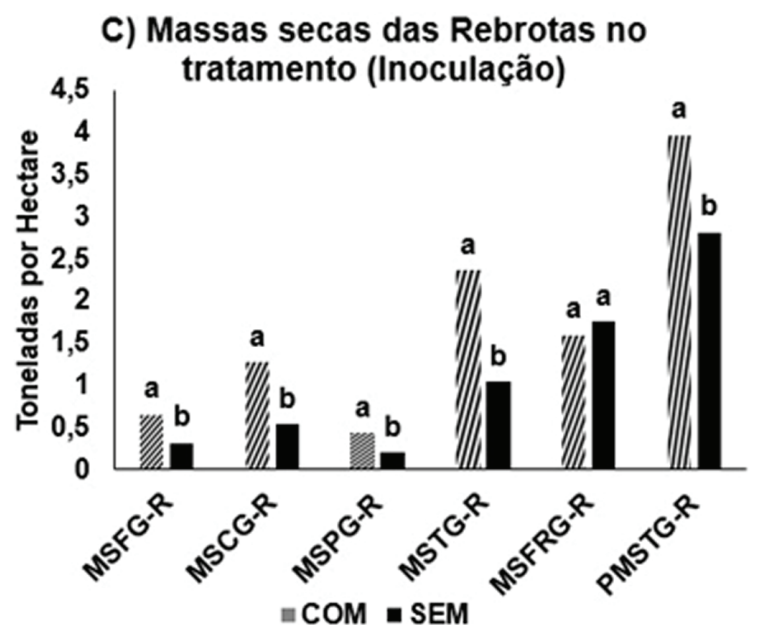

\begin{tabular}{lrr}
\hline Fator & \multicolumn{1}{c}{$\mathrm{Fc}$} & $\mathrm{Pr}>\mathrm{Fc}$ \\
\hline Ano (MSFG-R) & 45,32 & 0,001 \\
\hline Ano (MSCG-R) & 65,07 & 0,001 \\
\hline Ano (MSPG-R) & 27,26 & 0,001 \\
\hline Ano (MSTG-R) & 57,61 & 0,001 \\
\hline Ano (MSFRG-R) & 0,28 & 0,6 \\
\hline Ano (PMSTG-R) & 21,83 & 0,001 \\
\hline
\end{tabular}

Figura 2. Produtividade de matéria seca de folhas (MSFG-R), colmo (MSCG-R), panícula (MSPG-R), total (MSTG-R) do sorgo granífero, do capim-paiaguás (MSFRG-R) e produtividade total (PMSTG-R) das rebotas. A) Ano, B) Capim e C) Inoculação. Selvíria-MS. 
Tabela 1. Desdobramento das interações significativas ano x consórcio ou não com capim-paiaguás, para produtividade de matéria seca de folhas (MSFG-R) e total das rebrotas do sorgo granífero (MSTG-R). SelvíriaMS.

\begin{tabular}{|c|c|c|}
\hline \multirow{2}{*}{ Tratamento } & \multicolumn{2}{|c|}{ Capim-paiaguás } \\
\hline \multirow{2}{*}{\multicolumn{3}{|c|}{$\begin{array}{l}\text { Com } \\
\qquad \text { MSFG-R }\left(\mathrm{t} \mathrm{ha}^{-1}\right)\end{array}$}} \\
\hline & & \\
\hline $1^{\circ}$ Ano & $0,11 b$ & $0,24 b$ \\
\hline $2^{\circ}$ Ano & $0,48 \mathrm{aB}$ & $1,13 \mathrm{aA}$ \\
\hline \multicolumn{3}{|c|}{ MSTG-R $\left(\mathrm{t} \mathrm{ha}^{-1}\right)$} \\
\hline $1^{\circ}$ Ano & $0,49 \mathrm{bB}$ & $1,13 \mathrm{bA}$ \\
\hline $2^{\circ}$ Ano & $1,83 \mathrm{aB}$ & $3,40 \mathrm{aA}$ \\
\hline
\end{tabular}

Médias seguidas de letras distintas minúscula na coluna e maiúscula na linha diferem entre si, pelo teste de Tukey a $5 \%$ de probabilidade.

Tabela 2. Desdobramento das interações significativas anos x inoculação por $A$. brasilense, para produtividade de matéria seca de folhas (MSFG-R), colmos (MSCG-R), panículas (MSPG-R) e total (MSTG-R) das rebrotas do sorgo granífero. Selvíria-MS.

\begin{tabular}{|c|c|c|}
\hline \multirow{3}{*}{ Tratamento } & \multicolumn{2}{|c|}{ Inoculação } \\
\hline & Com & Sem \\
\hline & \multicolumn{2}{|c|}{ MSFG-R $\left(\mathrm{t} \mathrm{ha}^{-1}\right)$} \\
\hline $1^{\circ}$ Ano & $0,24 b$ & $0,11 b$ \\
\hline $2^{\circ}$ Ano & $1,09 \mathrm{aA}$ & $0,52 \mathrm{aB}$ \\
\hline \multicolumn{3}{|c|}{ MSCG-R (t ha $\left.{ }^{-1}\right)$} \\
\hline $1^{\circ}$ Ano & $0,61 \mathrm{bA}$ & $0,29 \mathrm{bB}$ \\
\hline $2^{\circ}$ Ano & $1,92 \mathrm{aA}$ & $0,78 \mathrm{aB}$ \\
\hline \multicolumn{3}{|c|}{ MSPG-R (t ha-1) } \\
\hline $1^{\circ}$ Ano & $0,24 b$ & $0,18 \mathrm{~b}$ \\
\hline $2^{\circ}$ Ano & $0,63 \mathrm{aA}$ & $0,28 \mathrm{aB}$ \\
\hline \multicolumn{3}{|c|}{ MSTG-R (t ha $\left.{ }^{-1}\right)$} \\
\hline $1^{\mathrm{o}}$ Ano & $1,10 \mathrm{bA}$ & $0,52 \mathrm{bB}$ \\
\hline $2^{\circ}$ Ano & $3,64 \mathrm{aA}$ & $1,58 \mathrm{aB}$ \\
\hline
\end{tabular}

Médias seguidas de letras distintas minúscula na coluna e maiúscula na linha diferem entre si, pelo teste de Tukey a 5\% de probabilidade. 
A) Massas secas das Rebrotas no tratamento (Ano)

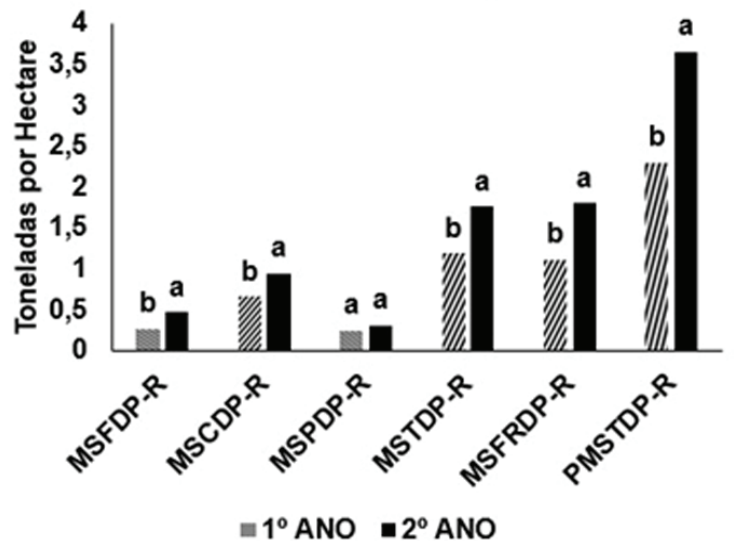

B) Massas secas das Rebrotas no tratamento (Capim)

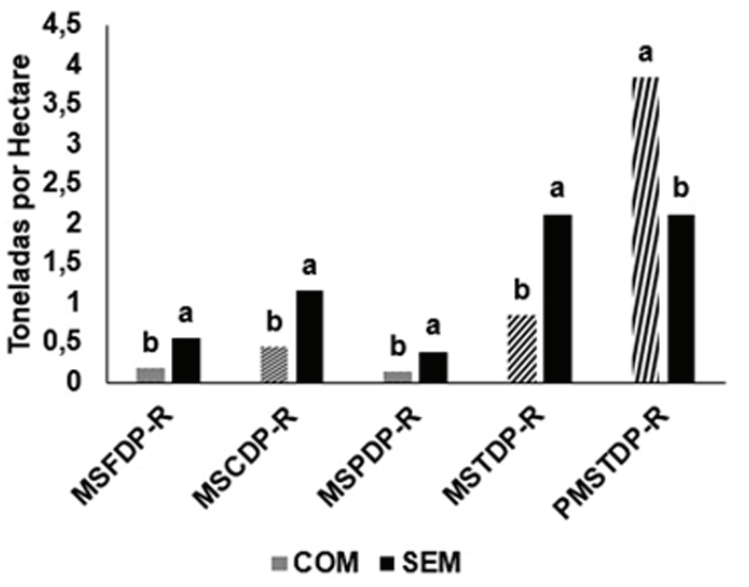

C) Massas secas das Rebrotas no tratamento (Inoculação)

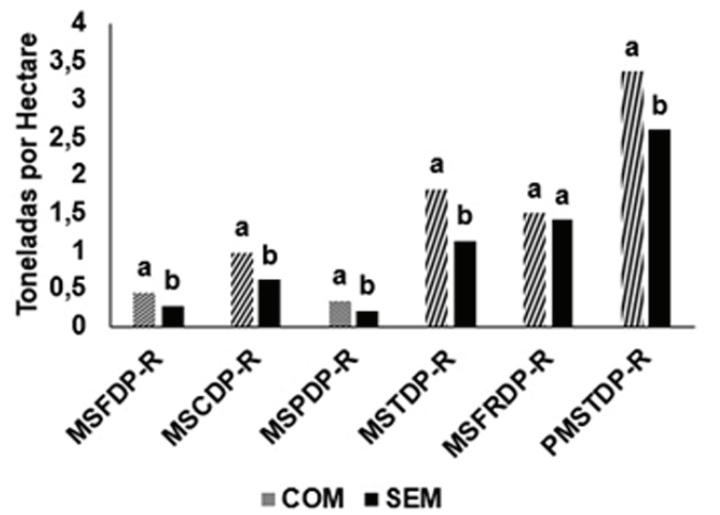

\begin{tabular}{lrr}
\hline Fator & \multicolumn{1}{c}{$F c$} & Pr>Fc \\
\hline Ano (MSFDP-R) & 56,33 & 0,001 \\
\hline Ano (MSCDP-R) & 9,46 & 0,001 \\
\hline Ano (MSPDP-R) & 1,12 & 0,3 \\
\hline Ano (MSTDP-R) & 21,72 & 0,001 \\
\hline Ano (MSFRDP-R) & 14,63 & 0,001 \\
\hline Ano (PMSTDP-R) & 29,06 & 0,001 \\
\hline
\end{tabular}

\begin{tabular}{lll}
\hline Fator & $\mathrm{Fc}$ & $\mathrm{Pr}>\mathrm{Fc}$ \\
\hline Ano (MSFDP-R) & 206,9 & 0,001 \\
\hline Ano (MSCDP-R) & 58,42 & 0,001 \\
\hline Ano (MSPDP-R) & 12,21 & 0,001 \\
\hline Ano (MSTDP-R) & 85,62 & 0,001 \\
\hline Ano (PMSTDP-R) & 47,2 & 0,001 \\
\hline
\end{tabular}

\begin{tabular}{lll}
\hline Fator & FC & PrPFC \\
\hline Ano (MSFDP-R) & 44,06 & 0,001 \\
\hline
\end{tabular} \begin{tabular}{lrr} 
Ano (MSCDP-R) & 15,25 & 0,001 \\
\hline Ano (MSPDP-R) & 4,52 & 0,042
\end{tabular} \begin{tabular}{lrr}
\hline Ano (MSPDP-R) & 4,52 & 0,042 \\
\hline Ano
\end{tabular} \begin{tabular}{lrr} 
Ano (MSTDP-R) & $29,62 \quad 0,001$ \\
\hline Ano
\end{tabular} \begin{tabular}{lll}
\hline Ano (MSFRDP-R) & $0,29 \quad 0,59$ \\
\hline Ano (PMSPP-R & 9,2 & 0,001
\end{tabular} \begin{tabular}{lrr}
\hline Ano (PMSTDP-R) & 9,42 & 0,001 \\
\hline
\end{tabular}

Figura 3. Produtividade de matéria seca de folhas (MSFDP-R), colmo (MSCDP-R), panícula (MSPDP-R), total (MSTDP-R) do sorgo de dupla aptidão, do capim-paiaguás (MSFRDP-R) e produtividade total das rebotas (PMSTDP-R). A) Ano, B) Capim e C) Inoculação. Selvíria-MS. 
sa, fato observado no primeiro ano de condução do experimento.

A produtividade de matéria seca do sorgo de dupla aptidão no consórcio com capim-paiaguás é alterada pela competição entre as espécies, no entanto, a produtividade total das plantas em consórcio (PMSTDP-R) é maior quando somada a matéria seca dos rebrotes de sorgo e capim, deixando no local quantidades satisfatórias de palhada para liberação de nutrientes e retenção de umidade como no caso particular do trabalho de Costa et al. (2015), em que a cobertura morta foi o fator determinante na diminuição da evapotranspiração.

Observa-se que as produtividades de matéria seca do sorgo e/ou capim, quando o sorgo foi inoculado por Azospirillum brasilense, aumentaram significativamente, entretanto, sem efeito para MSFRDP-R. Nesta mesma linha de pesquisa, Nakao et al. (2018) verificaram aumentos significativos para a produção de massa seca total de sorgos e forrageira em consórcio com a inoculação de bactérias do gênero Azospirillum. Esses resultados evidenciam a importância das bactérias nas culturas produtoras de forragem e grãos, no aumento da produção, uma das hipóteses é o aumento do volume de raízes, o que melhora a capacidade de a planta obter água e nutrientes ou que tenha maior eficiência no seu uso, resistindo melhor à seca.

Para o desdobramento das interações ano $\mathrm{x}$ consórcio ou não com capim para MSFDP-R e MSTDP-R (Tabela 3), constatou-se que, com a ausência do consórcio, as produtividades destas duas componentes (MSFDP-R e MSTDP-R) tendem a aumentar, resultado que pode ser atribuído ao efeito de competição das plantas em consórcio.

Para o acúmulo de nutrientes na rebrota do sorgo granífero (Figura 4A), verifica-se que houve resposta significativa dos anos de condução, em que mesmo após o primeiro ano do experimento e retirada de material vegetal para ensilagem, a rebrota acumulou maiores quantidades de nutrientes, apresentando grande incremento de palhada para a continuidade do SPD. Assim, é válido o uso desse sistema em reciclar nutrientes durante a permanência da palhada e possibilitando incrementar a produtividade de grãos em sucessão.

Tabela 3. Desdobramento das interações significativas ano x capim-paiaguás, para a produtividade de matéria seca de folhas (MSFDP-R) e total (MSTDP-R) das rebrotas do sorgo de dupla aptidão. Selvíria-MS.

\begin{tabular}{|c|c|c|}
\hline \multirow{3}{*}{ Tratamento } & \multicolumn{2}{|c|}{ Capim-paiaguás } \\
\hline & Com & Sem \\
\hline & \multicolumn{2}{|c|}{ MSFDP-R $\left(\mathrm{t} \mathrm{ha}^{-1}\right)$} \\
\hline $1^{\circ}$ Ano & $0,14 \mathrm{~B}$ & $0,40 \mathrm{bA}$ \\
\hline \multirow[t]{2}{*}{$2^{\circ}$ Ano } & $0,21 B$ & $0,73 \mathrm{aA}$ \\
\hline & \multicolumn{2}{|c|}{$\operatorname{MSTDP}-\mathrm{R}\left(\mathrm{t} \mathrm{ha}^{-1}\right)$} \\
\hline $1^{\circ}$ Ano & $0,70 \mathrm{~B}$ & $1,67 \mathrm{bA}$ \\
\hline $2^{\circ}$ Ano & $0,97 \mathrm{~B}$ & $2,56 \mathrm{aA}$ \\
\hline
\end{tabular}


Para o consórcio sorgo/capim-paiaguás (Figura 4B), houve um aumento no acúmulo de N, P, K, Ca e $\mathrm{Mg}$. Isso pode ser devido principalmente à maior produção de matéria seca das rebrotas em consorciação (Figura 2B). Do mesmo modo, quando houve a inoculação por $A$. brasilense, aumentaram-se os acúmulos de N, P, Ca e Mg (Figura 4C), uma vez que as produções de matéria seca também foram superiores quando houve a inoculação nas sementes de sorgo granífero (Figura 2C).

Deve-se ressaltar também que nas áreas destinadas à produção de silagem são retiradas grandes quantidades de nutrientes, o que pode gerar deficiência no solo. Com a ausência de resíduos, o teor de matéria orgânica no solo pode ser reduzido, bem como a redução da capacidade de retenção de água, o que leva a uma condição de maior compactação, prejudicando o estabelecimento da cultura subsequente, com resultados negativos na produtividade final.

As rebrotas dos sorgos consorciados com capim-paiaguás podem ajudar a minimizar os efeitos negativos sobre o solo dessas áreas de cultivo, principalmente na reposição de material orgânico. Foram verificados resultados positivos dos consórcios, evidenciando os benefícios das forrageiras na ciclagem de nutrientes.

Além disso, esta tecnologia não apresenta dificuldades para emissão de rebrotas desde que haja o manejo e a sistematização correta na área a ser cultivada. Assim, haverá uma produtividade satisfatória das rebrotas em consórcio, principalmente com a inclusão do capim que atenderá um dos requisitos básicos para a sustentabilidade do SPD, a cobertura permanente do solo, garantindo estabilidade econômica para o produtor.

$\mathrm{O}$ acúmulo de nutrientes da rebrota do sorgo de dupla aptidão (Figura 5A) assemelhou-se à varia- ção dos nutrientes nos anos de condução, do mesmo modo que para o granífero (Figura 4A), com aumento significativo para $\mathrm{N}, \mathrm{P}, \mathrm{K}, \mathrm{Ca}$ e $\mathrm{Mg}$ no segundo ano. $\mathrm{O}$ acúmulo de $\mathrm{S}$ para os dois tipos de sorgo avaliados não apresentou aumento entre anos de avaliação, como também na consorciação com o capim-paiaguás e inoculação pela bactéria. De acordo com Mateus et al. (2011), estudando o sorgo-de-guiné (Sorghum bicolor subespécie bicolor raça guinea), houve também alta produção de matéria seca e ciclagem de N, P e K.

Houve incrementos de nutrientes por ocasião da consorciação do capim-paiaguás nos resíduos das rebrotas das culturas (Figura 5B). Em condições de adequado suprimento de água (segundo ano - Figura 1), condições estas que podem ocorrer esporadicamente em regiões de inverno seco no Cerrado, o cultivo consorciado pode fornecer cobertura vegetal e nutrientes ao solo, mostrando-se uma opção viável mesmo com a competição das duas espécies em consórcio.

A inoculação do sorgo por $A$. brasilense influencia no aumento da sua área radicular melhorando a absorção de água e nutrientes (Okon \& Labandera-Gonzalez, 1994), podendo ser uma hipótese para o aumento da produtividade de matéria seca da rebrota e consequentemente da quantidade de nutrientes acumulados na parte aérea das plantas. De modo geral, o sorgo tem a característica de ser tolerante à seca em razão do seu maior volume radicular (Nour \& Welbel, 1978), conferindo às plantas maior eficiência de rebrota mesmo sob déficit hídrico, pelo maior comprimento das raízes.

Nas Tabelas 4 e 5 estão os resultados médios dos acúmulos de $\mathrm{K}$ nas interações dos anos de condução com ou sem capim-paiaguás, e inoculado ou não com $A$. brasilense, respectivamente. Verificouse elevada quantidade de K para as duas interações, com aumento no segundo ano, mesmo sem consórcio 


\section{A) Acúmulo de nutrientes na Rebrota}

(Ano)

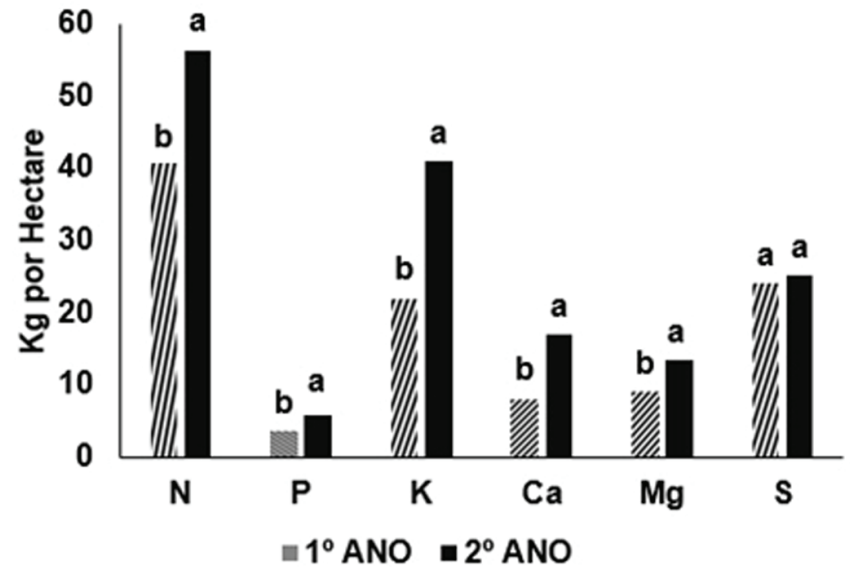

\begin{tabular}{lrr}
\hline Fator & \multicolumn{1}{l}{$\mathrm{Fc}$} & $\mathrm{Pr}>\mathrm{Fc}$ \\
\hline Ano $(\mathrm{N})$ & 19,24 & 0,001 \\
\hline Ano $(\mathrm{P})$ & 17,06 & 0,001 \\
\hline Ano $(\mathrm{K})$ & 57,32 & 0,001 \\
\hline Ano $(\mathrm{Ca})$ & 60,86 & 0,001 \\
\hline Ano $(\mathrm{Mg})$ & 10,8 & 0,003 \\
\hline Ano $(\mathrm{S})$ & 0,76 & 0,075 \\
\hline
\end{tabular}

B) Acúmulo de nutrientes na Rebrota

(Capim)

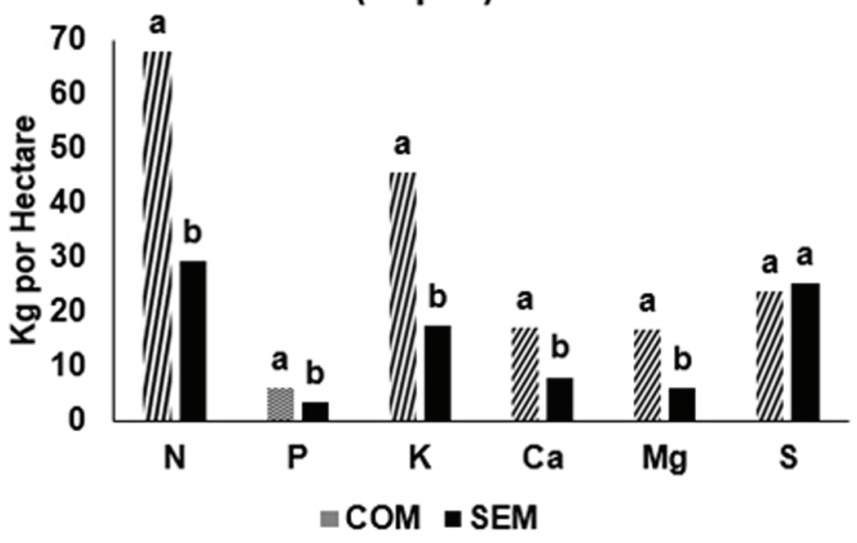

\begin{tabular}{lrr}
\hline Fator & \multicolumn{1}{c}{$\mathrm{FC}$} & $\mathrm{Pr}>\mathrm{FC}$ \\
\hline Capim $(\mathrm{N})$ & 99,42 & 0,001 \\
\hline Capim (P) & 23,22 & 0,001 \\
\hline Capim (K) & 99,85 & 0,001 \\
\hline Capim (Ca) & 62,9 & 0,001 \\
\hline Capim (Mg) & 62,83 & 0,001 \\
\hline Capim (S) & 2,2 & 0,120 \\
\hline
\end{tabular}

C) Acúmulo de nutrientes na Rebrota

(Inoculação)

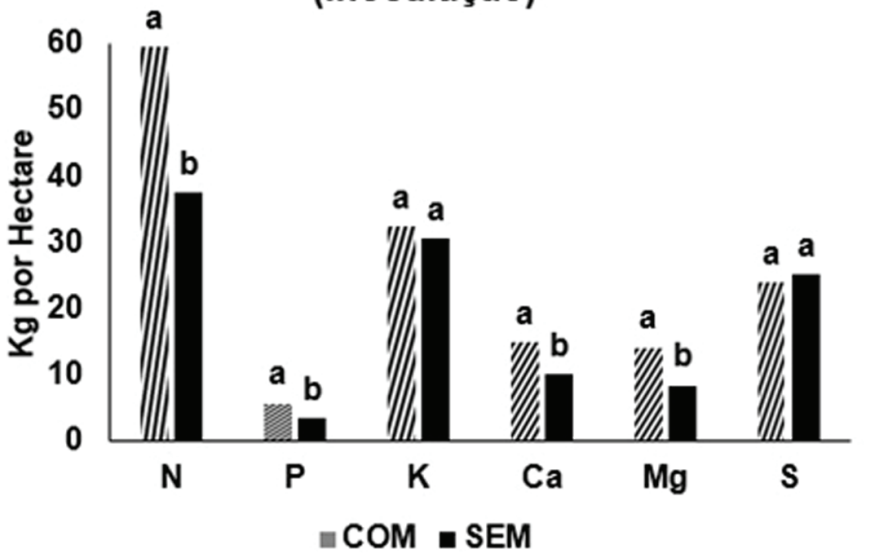

\begin{tabular}{lrr}
\hline Fator & \multicolumn{1}{c}{$\mathrm{FC}$} & $\mathrm{Pr}>\mathrm{Fc}$ \\
\hline Inoculação (N) & 38,27 & 0,001 \\
\hline Inoculação (P) & 19,1 & 0,001 \\
\hline Inoculação (K) & 0,56 & 0,462 \\
\hline Inoculação (Ca) & 19,12 & 0,002 \\
\hline Inoculação (Mg) & 17,37 & 0,001 \\
\hline Inoculação (S) & 1,01 & 0,952 \\
\hline
\end{tabular}

Figura 4. Acúmulos de $\mathrm{N}, \mathrm{P}, \mathrm{K}, \mathrm{Ca}, \mathrm{Mg}$ e $\mathrm{S}$ das rebrotas de sorgo granífero, em cultivo exclusivo ou em consórcio com o capim-paiaguás, durante dois anos agrícolas. A) Ano, B) Capim e C) Inoculação. Selvíria-MS. 
A) Acúmulo de nutrientes na Rebrota

(Ano)

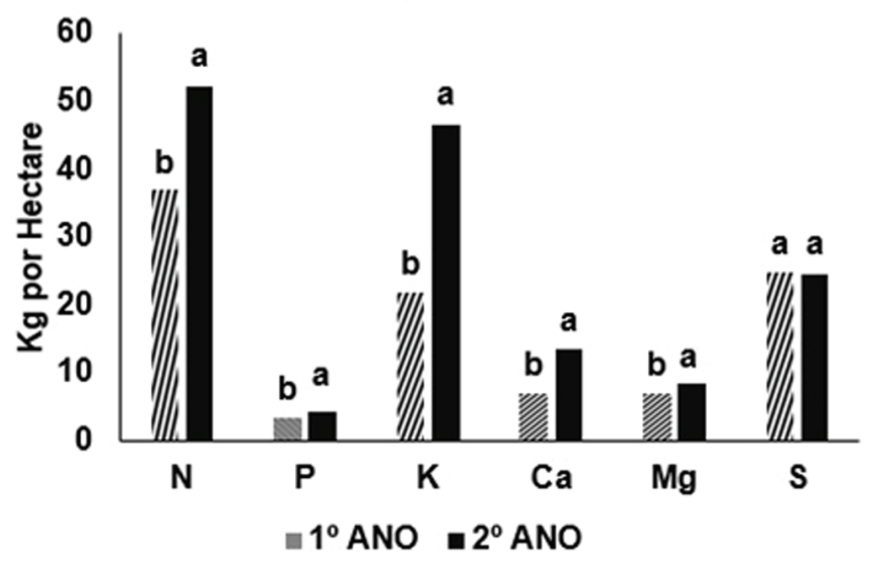

\begin{tabular}{lrr}
\hline Fator & \multicolumn{1}{l}{ Fc } & \multicolumn{1}{c}{ Pr> Fc } \\
\hline Ano $(\mathrm{N})$ & 15,94 & 0,001 \\
\hline Ano $(\mathrm{P})$ & 12,84 & 0,001 \\
\hline Ano $(\mathrm{K})$ & 79,23 & 0,001 \\
\hline Ano $(\mathrm{Ca})$ & 33,25 & 0,001 \\
\hline Ano $(\mathrm{Mg})$ & 4,9 & 0,038 \\
\hline Ano $(\mathrm{S})$ & 0,47 & 0,248 \\
\hline
\end{tabular}

B) Acúmulo de nutrientes na Rebrota

(Capim)

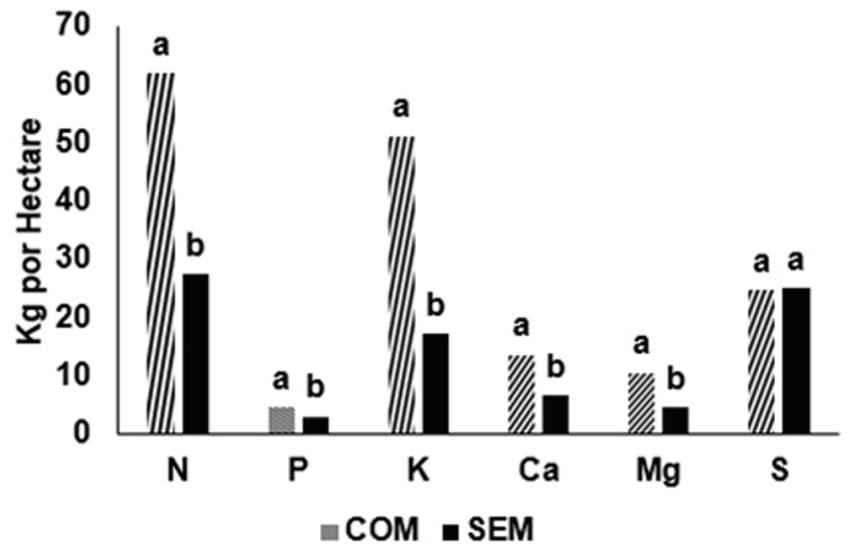

\begin{tabular}{lrr}
\hline Fator & \multicolumn{1}{l}{$\mathrm{FC}$} & $\mathrm{Pr}>\mathrm{FC}$ \\
\hline Capim $(\mathrm{N})$ & 82,91 & 0,001 \\
\hline Capim $(\mathrm{P})$ & 28,54 & 0,001 \\
\hline Capim $(\mathrm{K})$ & 99,13 & 0,001 \\
\hline Capim (Ca) & 37,19 & 0,001 \\
\hline Capim (Mg) & 79,14 & 0,001 \\
\hline Capim (S) & 0,57 & 0,258 \\
\hline
\end{tabular}

\section{C) Acúmulo de nutrientes na Rebrota} (Inoculação)

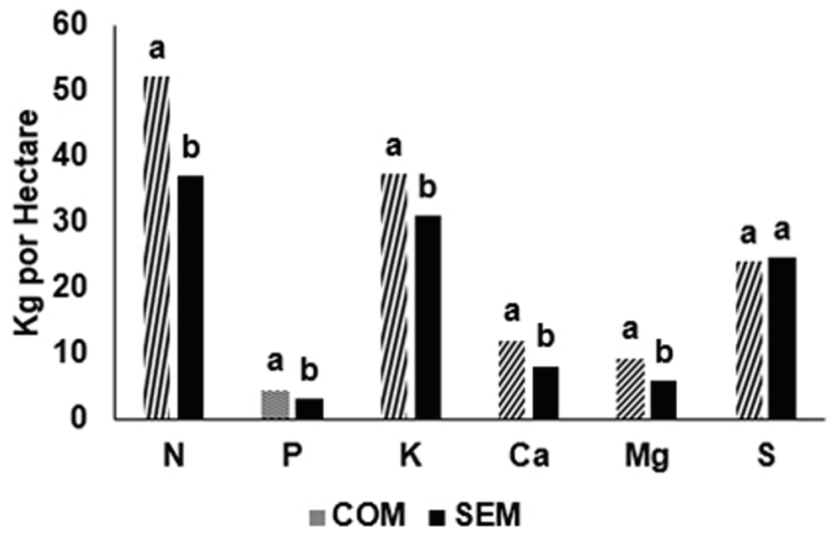

\begin{tabular}{lrr}
\hline Fator & \multicolumn{1}{c}{$\mathrm{FC}$} & $\mathrm{Pr}>\mathrm{FC}$ \\
\hline Inoculação (N) & 15,91 & 0,001 \\
\hline Inoculação (P) & 23,61 & 0,001 \\
\hline Inoculaçăo (K) & 5,54 & 0,028 \\
\hline Inoculação (Ca) & 11,90 & 0,002 \\
\hline Inoculação (Mg) & 29,09 & 0,001 \\
\hline Inoculação (S) & 1,61 & 0,151 \\
\hline
\end{tabular}

Figura 5. Acúmulos de N, P, K, Ca, Mg e $\mathrm{S}$ das rebrotas de sorgo de dupla aptidão, em cultivo exclusivo ou em consórcio com o capim-paiaguás, durante dois anos agrícolas. A) Ano, B) Capim e C) Inoculação. Selvíria-MS. 
Tabela 4. Desdobramento das interações significativas anos de cultivo x capim-paiaguás, para o acúmulo de K, durante dois anos agrícolas. Selvíria-MS.

\begin{tabular}{|c|c|c|}
\hline \multirow{2}{*}{ Tratamento } & \multicolumn{2}{|c|}{ Capim-paiaguás } \\
\hline & Com $\mathrm{K}\left(\mathrm{kg} \mathrm{ha}^{-1}\right)$ & Sem \\
\hline $1^{\circ}$ Ano & $33,39 \mathrm{bA}$ & $10,35 \mathrm{bB}$ \\
\hline $2^{\circ}$ Ano & $68,95 \mathrm{aA}$ & $24,21 \mathrm{aB}$ \\
\hline
\end{tabular}

Médias seguidas de letras distintas minúscula na coluna e maiúscula na linha diferem entre si, pelo teste de Tukey a $5 \%$ de probabilidade.

Tabela 5. Desdobramento das interações significativas anos de cultivo x inoculação por $A$. brasilense, para o acúmulo de K, durante dois anos agrícolas. Selvíria-MS.

\begin{tabular}{lccc}
\hline & Tratamento & Inoculação & Sem \\
& Com & $\mathrm{K}\left(\mathrm{kg} \mathrm{ha}^{-1}\right)$ & \\
$1^{\circ}$ Ano & $21,99 \mathrm{~b}$ & $21,75 \mathrm{~b}$ \\
$2^{\circ}$ Ano & $52,99 \mathrm{aA}$ & $40,16 \mathrm{aB}$ \\
& & \\
\hline
\end{tabular}

Médias seguidas de letras distintas minúscula na coluna e maiúscula na linha diferem entre si, pelo teste de Tukey a $5 \%$ de probabilidade.

e inoculação. Para o consórcio e inoculação dentro dos anos, fica nítida a importância dos consórcios e a inoculação no acúmulo de K pelo incremento na matéria seca produzida. Assim, consórcio e inoculação aumentam a reposição desse nutriente por meio das rebrotas das plantas, tendo em vista principalmente a complexidade destes sistemas produtivos, com alta taxa de exportação do $\mathrm{K}$ via silagem.

\section{Conclusões}

O aproveitamento da rebrota de sorgo granífero ou de dupla aptidão em consórcio com o capim-paiaguás apresentou-se viável para produção de palhada remanescente no período de entressafra, com maiores acúmulos de nutrientes.
A inoculação com Azospirillum brasilense nas sementes de sorgo granífero e de dupla aptidão proporciona maiores desenvolvimentos tanto das rebrotas do sorgo quanto do capim-paiaguás em consórcio.

\section{Referências}

ACOMPANHAMENTO DA SAFRA BRASILEIRA [DE] GRÃOS: safra 2017/18: segundo levantamento. Brasília, DF: Conab, 2017. v. 5, 125 p.

BOTELHO, P. R. F.; PIRES, D. A. A.; SALES, E. C. J.; ROCHA JÚNIOR, V. R.; JAYME, D. G.; REIS, S. T. Avaliação de genótipos de sorgo em primeiro corte e rebrota para produção de ensilagem. Revista Brasileira de Milho e Sorgo, Sete Lagoas, v. 9, n. 3, p. 287-297, 2010. DOI: 10.18512/1980-6477/rbms.v9n3p287-297. 
CONAB. Companhia Nacional de Abastecimento. Produção de sorgo cresce 11\%. Brasília, DF, 2011. Disponível em: $\quad<$ http://www.conab.gov.br/noticiaimprensa.php?id=22803>. Acesso em: 5 dez. 2011.

COSTA, E. J. B.; SOUZA, E. S.; BARROS JÚNIOR, G.; NUNES FILHO, J.; SOUZA, J. R.; TABOSA, J. N.; LEITE, M. L. M. V. Cultivo de sorgo em sistema de vazante com e sem cobertura do solo. Revista Brasileira de Milho e Sorgo, Sete Lagoas, v. 14, n. 2, p. 182-195, 2015. DOI: 10.18512/1980-6477/rbms.v14n2p182-195.

DARTORA, J.; GUIMARÃES, V. F.; MARINI, D.; SANDER, G. Adubação nitrogenada associada à inoculação com Azospirillum brasilense e Herbaspirillum seropedicae na cultura do milho. Revista Brasileira de Engenharia Agrícola e Ambiental, Campina Grande, v. 17, n. 10, p. 1023-1029, 2013.

DOI: 10.1590/S1415-43662013001000001.

DOBBELAERE, S.; CROONENBORGHS, A.; THYS, A.; PTACEK, D.; VANDERLEYDEN, J.; DUTTO, P.; LABANDERA-GONZALEZ, C.; CABALLERO-MELLADO, J.; AGUIRRE, J. F.; KAPULNIK, Y.; BRENER, S.; BURDMAN, S.; KADOURI, D.; SARIG, S.; OKON, Y. Responses of agronomically important crops to inoculation with Azospirillum. Australian Journal of Plant Physiology, East Melbourne, v. 28, n. 9, p. 871-879, 2001. DOI: 10.1071/PP01074.

FERREIRA, D. F. Sisvar: a guide for its bootstrap procedures in multiple comparisons. Ciência e Agrotecnologia, Lavras, v. 38, n. 2, p. 109-112, 2014.

DOI: $10.1590 / \mathrm{S} 1413-70542014000200001$.

FAO. Food and Agriculture Organization of the United Nations. Coarse grains. Roma, 2014. Disponível em: $<$ http://www.fao.org/docrep/010/ai466e/ai466e04.htm>. Acesso em: 1 abr. 2014.

HUNGRIA, M. Inoculação com Azospirillum brasiliense: inovação em rendimento a baixo custo. Londrina: Embrapa Soja, 2011. 36 p. (Embrapa Soja. Documentos, 325).
MALAVOLTA, E.; VITTI, G. C.; OLIVEIRA, S. A. Avaliação do estado nutricional das plantas: princípios e aplicações. 2. ed. Piracicaba: Associação Brasileira para Pesquisa da Potassa e do Fosfato, 1997.

MAGALHÃES, P. C.; DURÃES, F. O. M. Ecofisiologia da produção de sorgo. Sete Lagoas: Embrapa Milho e Sorgo, 2003. 4 p. (Embrapa Milho e Sorgo. Comunicado Técnico, 87).

MATEUS, G. P.; CRUSCIOL, C. A. C.; BORGHI, E.; PARIZ, C. M.; COSTA, C.; SILVEIRA, J. P. F. Adubação nitrogenada de sorgo granífero consorciado com capim em sistema de plantio direto. Pesquisa Agropecuária Brasileira, Brasília, DF, v. 46, n. 10, p. 1161-1169, 2011.

DOI: 10.1590/S0100-204X2011001000007.

NAKAO, A. H.; SOUZA, M. F. P.; DICKMANN, L.; CENTENO, D. C.; RODRIGUES, R. A. F. Resposta do sorgo granífero à aplicação de diferentes doses e épocas de inoculante (Azospirillum brasilense) via foliar. Enciclopédia Biosfera, Goiânia, v. 10, n. 18, p. 27022714, 2014.

NAKAO, A. H.; ANDREOTTI, M.; SOARES, D. A.; MODESTO, V. C.; DICKMANN, L. Intercropping Urochloa brizantha and sorghum inoculated with Azospirillum brasilense for silage. Revista Ciência Agronômica, Fortaleza, v. 49, n. 3, p. 501-511, 2018. DOI: $10.5935 / 1806-6690.20180057$.

NOUR, A. M.; WEIBEL, D. E. Evaluation of root characteristics in grain sorghum. Agronomy Journal, Madison, v. 70, n. 2, p. 217-218, 1978. DOI: 10.2134/agronj1978.00021962007000020002x.

OKON, Y.; LABANDERA-GONZALEZ, C. Agronomic application of Azospirillum: an evaluation of 20 years worldwide field incubation. Soil Biology and Biochemistry, Oxford, v. 26, n. 12, p. 1591-1601, 1994. DOI: 10.1016/0038-0717(94)90311-5.

RAIJ, B. van; ANDRADE, J. C.; CANTARELLA, H.; QUAGGIO, J. A. Análise química para avaliação 
da fertilidade de solos tropicais. Campinas: Instituto Agronômico, 2001. 284 p.

SANTOS, H. G.; JACOMINE, P. K. T.; ANJOS, L. H. C.; OLIVEIRA, V. A. V.; LUMBRERAS, J. F.; COELHO, M. R.; ALMEIDA, J. A.; CUNHA, T. J. F.; OLIVEIRA, J. B. Sistema brasileiro de classificação de solos. 3. ed. Brasília, DF: Embrapa-SPI, 2013. 353 p.

SILVA, A. G.; MORAES, L. E.; HORVATHY NETO, A.; TEIXEIRA, I. R.; SIMON, G. A. Consórcio na entrelinha de sorgo com braquiária na safrinha para produção de grãos e forragem. Semina: Ciências Agrárias, Londrina, v. 34, n. 6, p. 3475-3488, 2013. Suplemento 1.

DOI: 10.5433/1679-0359.2013v34n6Supl1p3475.

ZAGO, C. P. Cultura do sorgo para produção de silagem de alto valor nutritivo. In: SIMPÓSIO SOBRE NUTRIÇÃO DE BOVINOS, 4., 1991, Piracicaba. Anais... Piracicaba: FEALQ, 1991. p. 169-217. 